\title{
Stigma, Substance Use, and Help-Seeking Attitudes Among Rural and Urban Individuals
}

Zach A. Dschaak

Cindy L. Juntunen

University of North Dakota, cl.juntunen@UND.edu

How does access to this work benefit you? Let us know!

Follow this and additional works at: https://commons.und.edu/ehb-fac

Part of the Health and Physical Education Commons

\section{Recommended Citation}

Zach A. Dschaak and Cindy L. Juntunen. "Stigma, Substance Use, and Help-Seeking Attitudes Among Rural and Urban Individuals" (2018). Education, Health \& Behavior Studies Faculty Publications. 10. https://commons.und.edu/ehb-fac/10

This Article is brought to you for free and open access by the Department of Education, Health \& Behavior Studies at UND Scholarly Commons. It has been accepted for inclusion in Education, Health \& Behavior Studies Faculty Publications by an authorized administrator of UND Scholarly Commons. For more information, please contact und.commons@library.und.edu. 


\title{
Stigma, substance use, and help-seeking attitudes among rural and urban
}

\section{individuals}

Dschaak, Zachary A. Juntunen, Cindy L.

\begin{abstract}
The current study examined the differences between public stigma, self-stigma, substance use (i.e., alcohol and/or drugs), and attitudes toward psychological help-seeking among rural and urban individuals, and found meaningful differences in public stigma by alcohol use. Two hundred and sixty participants recruited via Amazon Mechanical Turk completed an online survey that included the Perceptions of Stigmatization by Others for Seeking Help scale, the Self-Stigma of Seeking Help scale, the Attitudes Toward Seeking Professional Psychological Help scale, the Alcohol Use Disorders Identification Test, the Drug Abuse Screening Test - 10, and demographics. The authors found significant between-group differences in public stigma for individuals who screened positive for an alcohol use disorder compared to those who used alcohol but did not meet the screening threshold. This finding suggested that there may be differences in stigmatization between individuals who only occasionally use alcohol and those with an alcohol use disorder. There were no significant differences in self-stigma or attitudes toward psychological help-seeking. Moreover, there were no significant between-group differences based on DAST-10 scores for individuals who did not report drug use, individuals who reported using drugs, and those who screened positive for a substance use disorder on public stigma, self-stigma, or attitudes toward psychological help-seeking. Contrary to the authors' hypothesis, the results did not demonstrate any significant differences between public stigma, self-stigma, or attitudes toward psychological help-seeking based on rurality (i.e., rural or urban).
\end{abstract}


The authors highlight areas for future research focus and considerations when further examining stigma, substance use, and help-seeking attitudes among rural and urban individuals.

Keywords: help-seeking, public stigma, rural, self-stigma, substance use

\section{Introduction}

During the last half-century, there has been an increase in research surrounding mental illness, particularly around identifying evidenced-based treatments and exploring barriers to treatment. In a report prepared for the Substance Abuse and Mental Health Services Administration, the Center for Behavioral Health Statistics and Quality (CBHSQ, 2016) found that over the last year, almost $18 \%$ of all adults (i.e., 43.4 million) living in the United States of America experienced a mental illness and an estimated 34.2 million received mental health care. These statistics do not include substance use disorders, which were estimated as occurring in 19.5 million American adults, with only $11.4 \%$ of these individuals receiving specialty substance use treatment over the last year (CBHSQ, 2016). Considering the number of individuals requiring mental health services and the low utilization rates of counseling, there is a need for researchers to further explore factors that promote and prevent the utilization of seeking mental health services.

Stigma is one of the most frequently cited factors that inhibit the utilization and continuation of mental health services (Corrigan, 2004). Corrigan identified two distinct but interacting types of stigma, public stigma (i.e., the general public's perception that the individual has an adverse trait or quality) and self-stigma (i.e., the internalization of public stigma). Research has shown that the perceptions of stigma surrounding psychological treatment predict attitudes toward seeking psychological help (Komiya, Good, \& Sherrod, 2000), intentions to 
seek psychological help (Rochlen, Mohr, \& Hargrove, 1999), and continuation of mental health services (Wade, Post, Cornish, Vogel, \& Tucker, 2011), as well as being associated with termination of treatment in older adults (Sirey et al., 2001). The majority of research on stigma has focused on mental health stigma, and there remains a notable lack of literature on the stigmatization of substance use and dependence (Adlaf, Hamilton, Wu, \& Noh, 2009; Janulis, Ferrari, \& Fowler, 2013). This dearth of research may reflect the higher levels of stigma associated with substance use and abuse. Generally, individuals who abuse substances report higher levels of stigma compared to individuals who have been hospitalized with a mental illness (Link, Struening, Rahav, Phelan, \& Nuttbrock, 1997). Ironically, the fact that addiction itself is stigmatized is sometimes assumed to prevent the use of substances, which may, in turn, serve to limit research into addiction and stigma (Rasinksi, Woll, \& Cooke, 2005). Recently, researchers have begun to provide support for the extension of the mental health stigma model onto the stigmatization of addiction (Janulis et al., 2013).

The public stigma associated with substance use and dependence could include negative attributions such as the individual who uses substances is dangerous, and the belief that the individual's illness is caused by his or her bad character (Link, Phelan, Bresnahan, Stueve, \& Pescosolido, 1999). Corrigan, Kuwabara, and O'Shaughnessy (2009) found that individuals with drug addictions are perceived as more blameworthy (i.e., more responsible for the development and cessation of their illness), dangerous, and feared compared to individuals with mental illnesses. Vogel, Wade, and Ascheman (2009) suggested that individuals in active drug addiction may experience more public stigma because of prevalent stereotypes about individuals addicted to substances. Researchers also have shown that there is a greater desire for social distance from individuals who abused substances compared to those with a mental illness (Corrigan et al., 
2009; Link et al., 1999). Individuals also may attempt to avoid the stigma associated with mental illness by not seeking mental health treatment (Corrigan, 2004). Moreover, the effects of public stigma on individuals who received mental health services include a loss of opportunity, social segregation, and loss of self-determination (Corrigan \& Shapiro, 2010). Finally, stigma and the subsequent discrimination was found to be associated with poor mental and physical health among drug users (Ahern, Stuber, \& Galea, 2007).

In addition to public stigma, self-stigma is the individual's internalization of public stigma (Corrigan, 2004) that causes a reduction in self-esteem and self-worth through selflabeling (Vogel, Wade, \& Haake, 2006). Typically, individuals will begin to internalize negative stereotypes about mental illnesses (e.g., individuals with a mental illness are worthless) before they themselves have been diagnosed with a mental illness (Link, 1987). However, few studies have examined the role of self-stigma among individuals who use substances (e.g., Luoma, Kulesza, Hayes, Kohlenberg, \& Larimer, 2014). Living in a society that stigmatizes mental illness could lower the self-esteem of individuals who received mental health services (Corrigan, 2004). This loss of self-esteem, and subsequent loss of self-efficacy promotes what Corrigan, Larson, and Rüsch (2009) described as the "why try effect", which occurs when self-stigma encourages individuals to believe they have nothing to offer and are defined by their illness. These individuals also may begin to think that they are unworthy of receiving mental health care (Corrigan, Larson, \& Rüsch, 2009). Furthermore, self-stigma has been shown to be a significant predictor of help-seeking attitudes and willingness to seek counseling (Vogel, Wade, \& Hackler, 2007). Similarly, the stigma experienced by individuals who use substances may prevent them from seeking treatment (Ahern et al., 2007).

Much of the extant stigma research has examined how stigma affects attitudes toward 
help-seeking and willingness to seek psychological services. Attitudes are a significant predictor of intentions and future behavior (Ajzen \& Fishbein, 1980). Corrigan (2004) asserted that an individual may not seek mental health services to avoid the negative label (i.e., label avoidance), which may be the most significant way in which stigma affects the utilization of mental health services. Vogel et al. (2007) discovered that, among undergraduates, self-stigma mediated the link between public stigma and willingness to seek counseling for psychological and interpersonal concerns. Furthermore, self-stigma was positively related to public stigma, and inversely related to positive help-seeking attitudes.

In addition to understanding the relationship between public stigma, self-stigma, and attitudes toward help-seeking, it is imperative that researchers consider these constructs in different environmental contexts. The prevalence of mental illness in rural areas is consistent with that of nonrural areas (Kessler et al., 1994), but access to services is not (Goldsmith, Wagenfeld, Manderscheid, \& Stiles, 1997). The population of a setting also is related to the level of stigma toward mental health (Hoyt, Conger, \& Valde, 1997). Kessler et al. (2001) found that individuals in rural areas were more likely to receive treatment for a mental illness and less likely to report that they experienced stigma. Contrary to Kessler et al.'s findings, Hammer, Vogel, and Heimerdinger-Edwards (2013) found evidence suggesting that stigma is more prominent among rural men compared to suburban and urban men. Consistent with these findings, Komiti, Judd, and Jackson (2006) discovered that individuals residing in a rural setting were less likely to seek psychological help from their general practitioner compared to urban individuals. Stewart, Jameson, and Curtin (2015) found higher levels of reported public stigma and self-stigma among older adults in rural communities compared to urban communities, but found no differences based on setting for reported willingness to utilize mental health services. 
Researchers have suggested that stigma may be more prominent in rural populations because of the lack of access to services and increased social visibility, which could exacerbate an individual's feelings of rejection, fear of ostracism, and promote label avoidance (Larson \& Corrigan, 2010). These inconsistencies may be a result of differences in methodology, measurement, and participant samples. Considering these mixed findings, there is a need for more examination into the constructs of public stigma, self-stigma, and attitudes toward psychological help-seeking among rural and urban individuals.

The intent of this study was to examine public stigma, self-stigma, substance use (i.e., alcohol and/or drugs), and attitudes toward psychological help-seeking among rural and urban individuals. Understanding these differences could lead to better-targeted intervention programming involving stigma reduction with the aim of promoting treatment utilization. In the current study, the first research question examined between-group differences in public stigma, self-stigma, and attitudes toward psychological help-seeking among different substance-using categories (non-use, use, screened positive for a substance use disorder) for alcohol and drugs. The authors hypothesized that individuals currently using alcohol and/or drugs would report higher levels of public stigma and self-stigma, and lower positive attitudes toward psychological help-seeking. The researchers also wanted to address Corrigan's et al. (2017) call for research around the examination of stigma in individuals who have a substance use disorder compared to those who use substances but do not meet criteria for a substance use disorder. Therefore, the authors examined differences between these groups. The second research question sought to answer whether there was a difference in self-reported public stigma, self-stigma, and attitudes toward psychological help-seeking among rural and urban individuals. The authors hypothesized that individuals from rural areas would endorse higher levels of public stigma and self-stigma 
and lower positive attitudes toward psychological help-seeking.

\section{Method}

\section{Participants}

The study sample included 260 participants recruited from Amazon Mechanical Turk (Mturk). The sample ranged in age from 20 to $68(M=38.80, S D=11.21)$. Regarding gender, $49.6 \%$ of the sample identified as men $(n=129)$ and $48.8 \%$ identified as women $(n=127)$. The majority of the sample identified as Caucasian $(78.5 \% ; n=204)$, with $7.7 \%$ identifying as Asian $(n=20), 5 \%$ as African American $(n=15)$, and $3.1 \%$ as Hispanic $(n=8)$. When considering sexual orientation, the sample identified as predominantly heterosexual ( $n=204 ; 90.4 \%)$, with $10(3.8 \%)$ identifying as bisexual, 4 as lesbian (1.5\%), and 3 as gay (1.2\%). Religious affiliation was diverse with $26.9 \%$ identifying as Christian-Protestant $(n=70), 21.9 \%$ as Agnostic $(n=$ 57), $14.6 \%$ as Christian-Catholic $(n=38)$, and $13.8 \%$ as Atheist $(n=36)$. Regarding education, $38.5 \%$ identified as having a Bachelor's degree $(n=100), 19.2 \%$ as having some college credit but not graduating $(n=50), 15.8 \%$ as having an Associate's or vocational degree $(n=41)$, and $13.8 \%$ as having a high school education or general equivalency diploma $(n=36)$. Income varied, with $18.1 \%$ reporting an income under $\$ 20,000(n=47), 33.1 \%$ between $\$ 20,000-$ 40,000 $(n=86)$, and $18.8 \%$ between $\$ 40,000-60,000(n=49)$ per year. Finally, $35 \%$ of the participants categorized their settings' level of rurality as being rural $(n=91)$ and $65 \%$ as urban $(n=169)$.

\section{Measures}

Perceptions of Stigmatization by Others for Seeking Help (PSOSH). The Perceptions of Stigmatization by Others for Seeking Help scale (Vogel et al., 2009) is a five-item self-report Likert-type survey that measures the perceived stigmatization by other people for seeking mental 
health services. After the instructions, "Imagine you had an emotional or personal issue that you could not solve on your own. If you sought counseling services for this issue, to what degree do you believe that the people you interact with would __", an individual responds based on the item with ranges of 1 (not at all) to 5 (a great deal). Sample items include, "React negatively to you" and "Think bad things of you." Scores are totaled with higher scores reflecting greater perceptions of public stigma. The PSOSH was shown to have good test-retest reliability at .82 and content validity was supported through moderate associations with three alternate stigma measures (Vogel et al., 2009). Internal consistency ranged between .79-.89 (Vogel et al., 2009). The internal consistency of the scores obtained for the current study was .94.

Self-Stigma of Seeking Help (SSOSH). The Self-Stigma of Seeking Help scale (Vogel et al., 2006) is a 10-item self-report Likert-type survey that measures the anticipated self-stigma an individual would experience for seeking mental health services. Items include, "I would feel inadequate if I went to a therapist for psychological help" and "It would make me feel inferior to ask a therapist for help." Items are rated on a five-point scale $(1=$ strongly disagree and $5=$ strongly agree), with five of the items being reverse-scored, and a higher score indicating greater self-stigma with receiving psychological help. Validity was supported via relationships with the Attitudes Toward Seeking Professional Psychological Help Scale $(r=-.63)$, and the Intention to Seek Counseling Inventory $(r=-.53)$ (Vogel et al., 2006). Internal consistency ranged from .86.91 (Vogel et al., 2006). The internal consistency of the scores obtained in the current sample was .94 .

Attitudes Toward Seeking Professional Psychological Help - Short Form (ATSPPH S). The Attitudes Toward Seeking Professional Psychological Help scale - Short Form (Fischer \& Farina, 1995) is a 10-item self-report Likert-type scale that examines personal attitudes toward 
receiving mental health services. Items include "If I believed I was having a mental breakdown, my first inclination would be to get professional attention" and "The idea of talking about problems with a psychologist strikes me as a poor way to get rid of emotional conflicts" (Fischer \& Farina, 1995). Items are rated on a four-point scale ranging from 0 (disagree) to 3 (agree), with five items being reverse-scored, and a higher score representing more positive attitudes toward seeking professional help. Internal consistency and test-retest correlation were good at .84 and .80 , respectively (Fischer \& Farina, 1995). The internal consistency of the scores obtained for the current study was .90 .

Alcohol Use Disorders Identification Test (AUDIT). The AUDIT, created by Saunders, Aasland, Babor, de la Fuente, and Grant (1993), is a 10-item self-report scale to assess hazardous consumption, alcohol dependence, and alcohol-related harm over the last 12 months. Each item is scored from 0 to 4 . Items include "How often do have a drink containing alcohol" and "How many drinks containing alcohol do you have on a typical day when you are drinking". A cutoff score of eight has been identified as a reference point for individuals who may be at risk for alcohol problems (Saunders et al., 1993). Test-retest reliability was good at .83 (Hays, Merz, \& Nicholas, 1995) and validity was supported through the ability to discriminate between hazardous and non-hazardous consumption (Saunders et al., 1993). The internal consistency of the scores obtained in the current sample was .87 .

Drug Abuse Screening Test (DAST-10). Created by Skinner (1982), the DAST-10 is a 10-item self-report survey assessing drug use (i.e., not including alcohol or tobacco use) related problems in the previous 12 months. Items include "Have you used drugs other than those required for medical reasons" and "Do you abuse more than one drug at a time". A cutoff score of three warrants further investigation and is likely to meet criteria for a substance use disorder 
(Skinner, 1982). The DAST-10 has been shown to have good internal consistency at .86 and strongly correlated (.97) with the DAST-20 (Cocco \& Carey, 1998). The internal consistency of the scores obtained for the current study was .73.

Demographics. Participants were asked to provide information on their gender, racial identity, sexual orientation, religious affiliation, level of education, and total household income. Furthermore, rurality (i.e., rural or urban) was measured by asking about the participants' subjective description of their hometown and current residence. These classifications were combined as there were no significant frequency differences between them. According to the United States Department of Agriculture (2017), the definition of rurality should be established by the purpose of the application. As this study examines attitudes and personal beliefs, participants were not given predetermined categories and instead were asked about their subjective description of their setting's level of rurality.

\section{Procedure}

Participants were recruited through Mturk, an online crowdsourcing platform in which individuals are paid to complete jobs called human intelligence tasks (HITs). Mturk workers have been found to be more diverse and reliable than the typical undergraduate population (Behrend, Sharek, Meade, \& Wiebe, 2011; Buhrmester, Kwang, \& Gosling, 2011). Mturk has been suggested as particularly useful for the recruitment of individuals with potential substance use problems because of the increased rates of screening positive for a substance use disorder (Shapiro, Chandler, \& Mueller, 2013). Furthermore, web-based research has been utilized to access difficult-to-reach research participants (e.g., stigmatized in the offline world; Mangan \& Reips, 2007). Researchers have recently utilized Mturk participants to conduct stigma research (e.g., Corrigan, Bink, Fokuo, \& Schmidt, 2015). The participants accessed the survey via their 
personal computer after reviewing the advertisement created by the authors. The survey was accessible to every Mturk worker who matched the authors' predetermined criteria.

Inclusion criteria included having an Mturk account (and identification number), being above the age of 18, currently residing in the United States of America, and having a designation of a "masters" worker. Amazon states that the masters designation "identifies high performing Workers ... who have demonstrated excellence across a wide range of HITs" (Amazon.com, 2017). After the workers reviewed the assent form, they completed the survey instruments and demographics. Participants were compensated $\$ 0.90$ (i.e., 10 cents per minute of estimated time to completion), which is considerably higher than the median hourly wage of $\$ 1.38$ for Mturk tasks (Horton \& Chilton, 2010). The survey time was estimated knowing that experienced Mturk workers complete surveys more quickly than the average university student (Kees, Berry, Burton, \& Sheehan, 2017).

To address methodological concerns with utilizing Mturk, the authors followed many of Chandler and Shapiro's (2016) recommendations. For example, we disguised the purpose of the study, conducted pre-screening early in the survey, prevented duplicate workers (by screening multiple worker identification numbers and IP addresses, and preventing ballot box stuffing within the Qualtrics survey platform), selected masters designated workers (i.e., for the high HIT acceptance ratio), and utilized quality attention checks (Chandler \& Shapiro, 2016). To screen for conscientious completion of the survey and verify quality data, the authors reviewed the Mturk Worker identification number, geolocation, survey duration, screener items, and correct input of the survey completion code. These assurance checks were used to remove double responses, confirm residence in the United States of America, and ensure quality data through diligent completion based on response times and item analysis. 


\section{Results}

Descriptive statistics for public stigma, self-stigma, substance use (i.e., alcohol and drugs), and attitudes toward psychological help-seeking among rural and urban individuals are reported in Table 1. Preliminary analyses determined that gender was significant at the .05 level with males reporting higher levels of public stigma $(F[3,254]=2.785, p=.041)$, self-stigma $(F[3,254]=2.878, \mathrm{p}=.037)$, more negative attitudes toward seeking professional psychological help $(F[3,254]=4.441, p=.005)$, and a higher AUDIT score $(F[3,254]=6.587, p=.000)$. There were no other significant group differences on public stigma, self-stigma, attitudes toward psychological help-seeking, or AUDIT and DAST-10 scores based on racial identity, sexual orientation, religious affiliation, education, or total household income.

Bivariate correlations (see Table 1) were conducted to review relationships between public stigma, self-stigma, attitudes toward psychological help-seeking, the AUDIT, and the DAST-10. Self-stigma had a moderate positive relationship with public stigma and a strong negative relationship with positive attitudes toward psychological help-seeking. Public stigma also had a weak negative relationship with attitudes toward seeking psychological help. Finally, AUDIT scores had a weak positive correlation to public stigma and DAST-10 scores.

\section{Hypotheses}

To test the first hypothesis that individuals currently using alcohol and/or drugs will report higher levels of public stigma and self-stigma, and lower positive attitudes toward helpseeking, two one-way between-groups analyses of variance (ANOVAs) were conducted. The first one-way between-groups ANOVA was conducted to compare differences based on AUDIT scores (i.e., non-use, use, screened positive for an alcohol use disorder) on public stigma, selfstigma, and attitudes toward psychological help-seeking. There was a significant association with 
AUDIT scores on public stigma $(F[2,257]=3.755, p=.025)$, but not on self-stigma $(F[2,257]$ $=.817, p=.443)$ or attitudes toward psychological help-seeking $(F[2,257]=.1 .486, p=.228)$. Post-hoc comparisons with Bonferroni correction found significant between-group differences on public stigma for individuals who used alcohol but did not screen positive for an alcohol use disorder $(M=8.32, S D=4.27)$ compared to those who $\operatorname{did}(M=10.39, S D=4.44)$.

The second one-way between-groups ANOVA was conducted to compare differences based on DAST-10 scores (i.e., non-use, use, screened positive for a substance use disorder) on public stigma, self-stigma, and attitudes toward psychological help-seeking. However, there were no significant group differences based on DAST-10 scores on public stigma $(F[2,257]=.887, p$ $=.413)$, self-stigma $(F[2,257]=.410, p=.410)$, or attitudes toward psychological help-seeking $(F[2,257]=.1 .667, p=.191)$.

To test the second hypothesis, that the level of rurality would be associated with levels of public stigma, self-stigma, and attitudes toward psychological help-seeking, a one-way betweengroups ANOVA was conducted. The authors' hypothesis that individuals from rural areas would endorse higher levels of public stigma and self-stigma and lower positive attitudes toward helpseeking was not supported. The one-way between-groups ANOVA found no significant differences based on rurality on public stigma $(F[1,258]=2.273, p=.133)$, self-stigma $(F[1$, $258]=0.020, p=.887)$, or attitudes toward psychological help-seeking $(F[1,258]=.299, p=$ $.585)$.

Based on these results, outside of public stigma among individuals who use alcohol, there appear to be no differences in public stigma, self-stigma, and attitudes toward psychological help-seeking among individuals who use drugs/and or alcohol and those who do not. Furthermore, the level of rurality did not appear to be significantly related to self-reported public 
stigma, self-stigma, and attitudes toward psychological help-seeking.

\section{Discussion}

The study examined the differences in public stigma, self-stigma, substance use (i.e., alcohol and/or drugs), and attitudes toward psychological help-seeking among rural and urban individuals. Consistent with previous research, (Vogel et al., 2007), the men in our sample also were more likely than woman to report higher levels of self-stigma along with lower positive attitudes toward seeking psychological help. The first hypothesis that individuals currently using alcohol and/or drugs will report higher levels of public stigma and self-stigma, and lower positive attitudes toward psychological help-seeking compared to individuals who do not use substances was partially supported. Post-hoc analyses identified a significant between-group difference in public stigma for individuals who screened positive for an alcohol use disorder compared to those who used alcohol but did not meet the screening threshold. Previous research has consistently shown that alcohol is less stigmatized than other substances (e.g., Parcesepe \& Cabassa, 2013). However, in this sample, individuals who screened positive for an alcohol use disorder perceived greater public stigma associated with receiving mental health services, than did individuals who used alcohol without meeting the screening threshold. Considering the stigmatization around addiction, it is not unexpected that an individual who may have an alcohol use disorder would perceive increased public stigma associated with seeking mental health services. This finding suggests that there may be differences in stigmatization for individuals who use alcohol and drugs that meet criteria for a substance use disorder compared to those who do not meet criteria. However, there were no significant group differences based on DAST-10 scores for individuals who did not report drug use, individuals who reported using drugs, and those who screened positive for a substance use disorder on public stigma, self-stigma, or 
attitudes toward psychological help-seeking. As Adlaf et al. (2009) suggested, maintaining stigmatizing attitudes may be difficult for an individual actively using substances. In essence, suppressing or avoiding stigmatizing thoughts around substance use would be adaptive for the individual. It also may be that stigma decreases as individuals develop a better understanding of addiction through their own experience.

Contrary to the authors' second hypothesis on rural and urban differences, there were no significant differences between rurality (i.e., rural or urban) on public stigma, self-stigma, or attitudes toward psychological help-seeking. This lack of differences contradicts recent studies that have found higher reported levels of stigma in rural populations compared to their urban counterparts (Hammer et al, 2013; Stewart, Jameson, \& Curtin, 2015). This may be attributed to the samples studied, as the researchers examined specific populations in men (Hammer et al., 2013) and older adults (Stewart, Jameson, \& Curtin, 2015). It also is possible that the differences between rural and urban populations, as it relates to stigma and attitudes toward psychological help-seeking, may not be as prevalent as previous research has suggested.

\section{Limitations}

The first limitation of this study was the lack of inquiry regarding previous mental health services or substance use treatments. Corrigan, Edwards, Green, Diwan, and Penn (2001) found that individuals who were more familiar with mental illness were less likely to endorse stigma. This may have caused individuals in our sample with previous mental health services to report lower levels of stigma. As the majority of extant stigma research has been conducted utilizing a college sample, the authors attempted to gather a community adult sample through utilizing Mturk. This sampling method potentially introduced a selection bias that likely influences the generalizability of the results. Although Amazon states there are 500,000 registered workers in 
190 countries (Amazon.com, 2017), researchers have suggested there are closer to 15,000 workers from the United States at a given time (Stewart et al., 2015). Furthermore, as with any data collected through self-report, there may have been a social desirability component (Lucas \& Baird, 2006), especially as it relates to the endorsement of stigma (for discussion see Corrigan \& Shapiro, 2010) and substance use. Additionally, as Mturk is a pay for performance system and the participants were paid above the median Mturk task pay, their motives for conscientious survey completion should be considered. However, Buhrmester et al. (2011) found that data quality is generally unchanged by compensation when workers in the United States are sampled and provide only self-report answers. This limitation is still important to consider when using an Mturk sample in which the participants may be selecting answers based on choices that they believe will assure payment or when they want their work accepted to keep up their work approval ratings (this limitation was brought to the attention of one of the authors when speaking with an Mturk worker whose work was rejected). Some researchers have also found uncharacteristically high levels of malingering among Mturk workers (Shapiro et al., 2013). Lastly, the author's decision to allow participants to self-determine rurality is another limitation. Future research should include more standardized definitions of rurality that include population and proximity to metropolitan areas (for an example see Rural-Urban Continuum Codes; U.S. Department of Agriculture Economic Research Services, 2003).

\section{Implications}

The results suggest a lack of differences in levels of public stigma, self-stigma, and helpseeking attitudes between individuals who use substances (alcohol and/or drugs) and those who do not. Our finding suggested that there may be differences in public stigma for individuals who only occasionally use alcohol and those with an alcohol use disorder. Researchers have found 
that exposure to public stigma may lead to self-stigma as individuals internalize the stigma and apply it to themselves (Corrigan, Watson, \& Barr, 2006) and make individuals less willing to seek psychological help (Vogel et al., 2007). Overall, given the lack of differences, interventions should continue to focus on the reduction of self-stigma to promote mental health service utilization, as it has been shown to mediate the relationship between public stigma and helpseeking attitudes (Vogel et al., 2007).Vogel and colleagues (2007) suggest that offering information in the form of educational programming, public workshops, and web-based information may assist individuals in identifying stigma and develop coping strategies, which may promote psychological help-seeking. Corrigan et al., suggested three agendas to reducing addiction stigma: services agenda, rights agenda, and the self-worth agenda. These interventions may be more generalizable across different contexts based on the lack of self-reported differences in our sample's stigma and help-seeking attitudes. Luoma, Kohlenberg, Hayes, and Fletcher (2012) also found success in promoting treatment adherence and reducing substance use through group-based interventions targeting shame through an acceptance and commitment paradigm. Future researchers should look to the extensive mental health stigma reduction literature for future addiction stigma reduction interventions (for discussion see Corrigan et al., 2017).

This study begins to address Corrigan's et al. (2017) request for research around the effects of stigmatization for individuals who have a substance use disorder compared to those who use substances without meeting diagnostic criteria. This study examined individuals who screened positive for alcohol use and drug use disorders but did not examine actual substance use disorder diagnoses and, as such, future research should examine these differences more thoroughly. The current study also examined the differences in self-reported stigma and attitudes 
toward psychological help-seeking based on substances used (i.e., alcohol and/or drugs).

However, this study only differentiated between alcohol and drugs (i.e., excluding tobacco), and therefore further analyses should be conducted to examine more specific substances and their effects on stigma and attitudes toward help-seeking. Finally, although attitudes are a predictor of future behavior (Ajzen \& Fishbein, 1980), future research should include a measure of actual help-seeking behavior.

The lack of difference in stigma and help-seeking attitudes based on rurality may also present some implications. Previous research examining differences between rural and urban communities often has found that rural community members experience higher levels of stigma and lower levels of positive help-seeking attitudes (Hammer et al, 2013; Stewart, Jameson, \& Curtin, 2015). Such research suggests that rural populations may require different types of interventions to reduce stigma and increase psychological help-seeking. However, the current findings suggest that developing new stigma-reduction strategies may not be necessary, at least for those rural residents with substance abuse concerns. The findings also may be consistent with Jameson and Blank's (2007) assertion that rurality is dimensional, rather than categorical. As such, future researchers should examine these constructs further while including other important variables such as proximity to urbanized areas, community supports, values, previous mental health treatment, and knowledge about mental illness and treatment services.

Finally, the sample collected in this study from Mturk also supports previous literature regarding the diversity of Mturk's worker pool (e.g., Behrend et al., 2011; Buhrmester et al., 2011) and offers implications for research. Future researchers may benefit from utilizing Mturk to access difficult to reach populations such as individuals who use and abuse substances. This is particularly important when using a homogeneous convenience sample may not be appropriate 
based on the research question and reaching a specific population is not feasible (e.g., in isolated rural areas).

\section{Conclusion}

Two important findings, both somewhat contrary to extant literature, are highlighted in this research. First, there are potential differences in public stigma related to alcohol use, an aspect of substance abuse that is frequently considered to be less stigmatizing. Second, rural and urban attitudes toward help-seeking and perceptions of stigma may both be more similar than has previously been assumed in past research and intervention planning. As described above, the implications of these findings are important for research, treatment, and public health interventions.

\section{References}

Adlaf, E. M., Hamilton, H. A., Wu, F., \& Noh, S. (2009). Adolescent stigma towards drug addiction: Effects of age and drug use behaviour. Addictive Behaviors, 34, 360-364. doi: 10.1016/j.addbeh.2008.11.012

Ahern, J., Stuber, J. \& Galea, S. (2007). Stigma, discrimination and the health of illicit drug users. Drug and Alcohol Dependence, 88, 188-196. doi:10.1016/j.drugalcdep.2006.10.014

Ajzen, I., \& Fishbein, M. (1980). Understanding attitudes and predicting social behavior. Englewood Cliffs, NJ: Prentice-Hall

Amazon Mechanical Turk. (2017, July 27). FAQS. Retrieved from https://requester.mturk.com/help/faq

Amazon Mechanical Turk. (2017, July 27). Requester tour. Retrieved from https://requester.mturk.com/tour

Behrend, T. S., Sharek, D. J., Meade, A. W., \& Wiebe, E. N. (2011). The viability of crowdsourcing for survey research. Behavior Research Methods, 43, 800-813. doi:10.3758/s13428-011-0081-0

Buhrmester, M., Kwang, T., \& Gosling, S. D. (2011). Amazon's Mechanical Turk: A new source of inexpensive, yet high-quality, data? Perspectives on Psychological Science, 6, 3-5. doi: $10.1177 / 1745691610393980$

Center for Behavioral Health Statistics and Quality. (2016). Key substance use and mental health indicators in the United States: Results from the 2015 National Survey on Drug Use and Health (HHS Publication No. SMA 16-4984, NSDUH Series H-51).

Chandler, J., \& Shapiro, D. (2016). Conducting clinical research using crowdsourced 
convenience samples. Annual Review of Clinical Psychology, 12, 53-81.

doi:10.1146/annurev-clinpsy-021815-093623

Cocco, K. M., \& Carey, K. B. (1998). Psychometric properties of the Drug Abuse Screening Test in psychiatric outpatients. Psychological Assessment, 10, 408-414.

Corrigan, P. (2004). How stigma interferes with mental health care. American Psychologist, 59, 614-625. doi:10.1037/0003-066X.59.7.614

Corrigan, P. W., Bink, A. B., Fokuo, J. K., \& Schmidt, A. (2015). The public stigma of mental illness means a difference between you and me. Psychiatry Research, 226, 186-191. doi:10.1016/j.psychres.2014.12.047

Corrigan, P. W., Edwards, A. B., Green, A., Diwan, S. L., \& Penn, D. L. (2001). Prejudice, social distance, and familiarity with mental illness. Schizophrenia Bulletin, 27, 219-225.

Corrigan, P. W., Kuwabara, S. A., \& O'Shaughnessy, J. (2009). The public stigma of mental illness and drug addiction: Findings from a stratified random sample. Journal of Social Work, 9, 139-147. doi:10.1177/1468017308101818

Corrigan, P. W., Larson, J. E., \& Rüsch, N. (2009). Self-stigma and the "why try" effect: Impact on life goals and evidence-based practices. World Psychiatry: Official Journal of the World Psychiatric Association, 8, 75-81

Corrigan, P. W., Schomerus, G., Shuman, V., Kraus, D., Perlick, D., Harnish, A., .. . Smelson, D. (2017). Developing a research agenda for reducing the stigma of addictions, part II: Lessons from the mental health stigma literature. American Journal on Addictions, 26, 67-74. doi: 10.1111/ajad.12436

Corrigan, P. W. \& Shapiro, J. R. (2010). Measuring the impact of programs that challenge the public stigma of mental illness. Clinical Psychology Review, 20, 907-922. doi:10.1016/j.cpr.2010.06.004

Corrigan, P. W., Watson, A. C., \& Barr, L. (2006). The self-stigma of mental illness: Implications for self-esteem and self-efficacy. Journal of Social and Clinical Psychology, 25, 875-884.

Fischer, E. H., \& Farina, A. (1995). Attitudes toward seeking professional psychological help: A shortened form and considerations for research. Journal of College Student Development,

Goldsmith, H. F., Wagenfeld, M. O., Manderscheid, R. W., \& Stiles, D. (1997). Specialty mental health services in metropolitan and nonmetropolitan areas: 1983 and 1990. Administration \& Policy in Mental Health, 24, 475-488.

Hammer, J. H., Vogel, D. L., \& Heimerdinger-Edwards, S. R. (2013). Men's help seeking: Examination of differences across community size, education, and income. Psychology of Men \& Masculinity, 14, 65-75. doi:10.1037/a0026813

Hays, R. D., Merz, J. F. and Nicholas, R. (1995). Response burden, reliability, and validity of the CAGE, Short MAST, and AUDIT alcohol screening measures. Behavioral Research Methods, Instruments \& Computers, 27, 277-280.

Horton, J. J., \& Chilton, L. B. (2010). The labor economics of paid crowdsourcing. Proceedings of the 11th ACM conference on Electronic commerce (pp. 209-218). New York, NY: ACM. doi:10.1145/1807342.1807376

Hoyt, D. R., Conger, R. D., Valde, J. G., \& Weihs, K. (1997). Psychological distress and help seeking in rural America. American Journal of Community Psychology, 25, 449-470.

Jameson, J. P., \& Blank, M. B. (2007). The role of clinical psychology in rural mental health services: Defining problems and developing solutions. Clinical Psychology: Science and Practice, 14, 283-298. doi:10.1111/j.1468-2850.2007.00089.x 
Janulis, P., Ferrari, J. R., \& Fowler, P. (2013). Understanding public stigma toward substance dependence. Journal of Applied Social Psychology, 43, 1065-1072. doi:10.1111/jasp.12070

Kees, J., Berry, C., Burton, S., \& Sheehan, K. (2017). An analysis of data quality: professional panels, student subject pools, and Amazon's mechanical turk. Journal of Advertising, 46, 141-155. doi:10.1080/00913367.2016.1269304

Kessler, R. C., Berglund, P. A., Bruce, M. L., Koch, R., Laska, E. M., Leaf, P. J., . . \& Wang, P. S. (2001). The prevalence and correlates of untreated serious mental illness. Health Services Research, 36, 987-1007.

Kessler, R. C., McGonagle, K. A., Zhao, S., Nelson, C. B., Hughes, M., Eshleman, S., . . \& \& Kendler, K. S. (1994). Lifetime and 12-month prevalence of DSM-III-R psychiatric disorders in the United States: Results from the National Comorbidity Survey. Archives of General Psychiatry, 51, 8-19. doi:10.1001/archpsyc.1994.03950010008002

Komiti, A., Judd, F., \& Jackson, H. (2006). The influence of stigma and attitudes on seeking help from a GP for mental health problems. Social Psychiatry \& Psychiatric Epidemiology, 41, 738-745. doi:10.1007/s00127-006-0089-4

Komiya, N., Good, G. E., \& Sherrod, N. B. (2000). Emotional openness as a predictor of college students' attitudes toward seeking psychological help. Journal of Counseling Psychology, 47, 138-143. doi: 10.1037/AJ022-0167.47.1.138

Larson, J. E., \& Corrigan, P. W. (2010). Psychotherapy for self-stigma among rural clients. Journal of Clinical Psychology, 66, 524-536. doi:10.1002/jclp.20679

Link, B. G. (1987). Understanding labeling effects in the area of mental disorders: An assessment of the effects of expectations of rejection. American Sociological Review, 52, 96-112.

Link, B. G., Phelan, J. C., Bresnahan, M., Stueve, A., \& Pescosolido, B. A. (1999). Public conceptions of mental illness: Labels, causes, dangerousness, and social distance. American Journal of Public Health, 89, 1328-1333.

Link, B.G., Struening, E. L., Rahav, M., Phelan, J. C., \& Nuttbrock, L. (1997). On stigma and its consequences: Evidence from a longitudinal study of men with dual diagnoses of mental illness and substance abuse. Journal of Health and Social Behavior, 38, 177-190.

Luoma, J. B., Kohlenberg, B. S., Hayes, S. C., \& Fletcher, L. (2012). Slow and steady wins the race: A randomized clinical trial of acceptance and commitment therapy targeting shame in substance use disorders. Journal of Consulting and Clinical Psychology, 80, 43-53. doi:10.1037/a0026070

Lucas, R. E., \& Baird, B. M. (2006). Global self-assessment. In M. Eid \& E. Diener (Eds.), Handbook of multimethod measurement in psychology (pp. 29-42). Washington, DC: American Psychological Association. doi:10.1037/11383-003

Luoma, J. B., Kulesza, M., Hayes, S. C., Kohlenberg, B., \& Larimer, M. (2014). Stigma predicts residential treatment length for substance use disorder. The American Journal of Drug and Alcohol Abuse, 40, 206-212. doi:10.3109/00952990.2014.901337

Mangan, M. A., \& Reips, U. (2007). Sleep, sex, and the web: Surveying the difficult-to-reach clinical population suffering from sexsomnia. Behavior Research Methods, 39, 233-236.

Parcesepe, A. M., \& Cabassa, L. J. (2013). Public stigma of mental illness in the United States: A systematic literature review. Administration and Policy in Mental Health and Mental Health Services Research, 40, 384-399. doi:10.1007/s10488-012-0430-z

Rasinksi, K. A., Woll, P., \& Cooke, A. (2005). Stigma and substance use disorders. In P. W. 
Corrigan (Ed.), On the stigma of mental illness: Practical strategies for research and social change (pp. 219-236). Washington, DC: American Psychological Association.

Rochlen, A. B., Mohr, J. J., \& Hargrove, B. K. (1999). Development of the attitudes toward career counseling scale. Journal of Counseling Psychology, 46, 196-206.

Saunders, J. B., Aasland, O. G., Babor, T. F., de la Fuente, J. R., \& Grant, M. (1993).

Development of the alcohol use disorders identification test (AUDIT): WHO collaborative project on early detection of persons with harmful alcohol consumption-II. Addiction, 88, 791-804. doi:10.1111/j.1360-0443.1993.tb02093.x

Shapiro, D. N., Chandler, J., \& Mueller, P. A. (2013). Using mechanical turk to study clinical populations. Clinical Psychological Science, 1, 213-220. doi:10.1177/2167702612469015

Sirey, J., Bruce, M. L., Alexopoulos, G. S., Perlick, D. A., Raue, P., Friedman, S. J, \& Meyers, B. S. (2001). Perceived stigma as a predictor of treatment discontinuation in young and older outpatients with depression. American Journal of Psychiatry, 158, 479-481.

Skinner, H. A. (1982). The Drug Abuse Screening Test. Addictive Behavior, 7, 363-371.

Stewart, H., Jameson, J. P., \& Curtin, L. (2015). The relationship between stigma and selfreported willingness to use mental health services among rural and urban older adults. Psychological Services, 12, 141-148. doi:10.1037/a0038651

Stewart, N., Ungemach, C., Harris, A., Bartels, D. M., Newell, B. R., Paolacci, G., \& Chandler, J. (2015). The average laboratory samples a population of 7,300 Amazon Mechanical Turk workers. Judgment and Decision Making, 10, 479-491.

United States Department of Agriculture. (2017, December 8). Measuring rurality. Retrieved from https://www.ers.usda.gov/topics/rural-economy-population/ruralclassifications/what-is-rural/

United States Department of Agriculture Economic Research Service. (2003). Measuring rurality: Rural-urban continuum codes. Retrieved from https://wayback.archiveit.org/5923/20110913215735/http://www.ers.usda.gov/Briefing/Rurality/RuralUrbCon/

Vogel, D. L., Wade, N. G., \& Ascheman, P. L. (2009). Measuring perceptions of stigmatization by others for seeking psychological help: Reliability and validity of a new stigma scale with college students. Journal of Counseling Psychology, 56, 301-308. doi:10.1037/a0014903

Vogel, D. L., Wade, N. G., \& Haake, S. (2006). Measuring the self-stigma associated with seeking psychological help. Journal of Counseling Psychology, 53, 325-337. doi:10.1037/0022-0167.53.3.325

Vogel, D. L., Wade, N. G., \& Hackler, A. H. (2007). Perceived public stigma and the willingness to seek counseling: The mediating roles of self-stigma and attitudes towards counseling. Journal of Counseling Psychology, 54, 40-50. doi:10.1037/0022-0167.54.1.40

Wade, N. G., Post, B. C., Cornish, M. A., Vogel, D. L., \& Tucker, J. R. (2011). Predictors of the change in self-stigma following a single session of group counseling. Journal of Counseling Psychology, 58, 170-182. doi:10.1037/a0022630 
Table 1.

Means, Standard Deviations, and Intercorrelations among Study Variables $(N=260)$

\begin{tabular}{|c|c|c|c|c|c|c|c|c|c|c|c|}
\hline \multirow[b]{3}{*}{ Scale } & \multirow[b]{3}{*}{1.} & \multirow[b]{3}{*}{2.} & \multirow[b]{3}{*}{3.} & \multirow[b]{3}{*}{4.} & \multirow[b]{3}{*}{5.} & \multicolumn{2}{|c|}{ Total } & \multicolumn{2}{|c|}{ Rural } & \multicolumn{2}{|c|}{ Urban } \\
\hline & & & & & & $(N=$ & 60) & $(N=$ & & $(N=$ & 69) \\
\hline & & & & & & $\mathrm{M}$ & SD & M & SD & $\mathrm{M}$ & SD \\
\hline 1. PSOSH & & & & & & 8.80 & 4.39 & 8.24 & 4.14 & 9.10 & 4.51 \\
\hline 2. SSOSH & $.64 * *$ & & & & & 24.54 & 9.44 & 24.43 & 8.80 & 24.60 & 9.80 \\
\hline 3. ATSPPH & $-.47 * *$ & $-.75 * *$ & & & & 17.75 & 7.07 & 18.08 & 6.89 & 17.57 & 7.18 \\
\hline 4. AUDIT & $.18^{* *}$ & .05 & -.07 & & & 3.89 & 5.05 & & & & \\
\hline 5. DAST-10 & .08 & .05 & -.06 & $.31 * *$ & & 1.58 & 1.50 & & & & \\
\hline
\end{tabular}

Note. PSOSH $=$ Perceptions of Stigmatization by Others for Seeking Help; SSOSH = Self-Stigma of Seeking Help; ATSPPH = Attitudes Toward Seeking Professional Psychological Help; AUDIT $=$ Alcohol Use Disorders Identification Test; and DAST $-10=$ Drug Abuse Screening Test -10 **. Correlation is significant at the 0.01 level (2-tailed). 